\title{
Special symmetries of Banach spaces isomorphic to Hilbert spaces
}

\author{
by \\ JARno TALPONEn (Aalto)
}

\begin{abstract}
We characterize Hilbert spaces among Banach spaces in terms of transitivity with respect to nicely behaved subgroups of the isometry group. For example, the following result is typical: If $\mathrm{X}$ is a real Banach space isomorphic to a Hilbert space and convex-transitive with respect to the isometric finite-dimensional perturbations of the identity, then $\mathrm{X}$ is already isometric to a Hilbert space.
\end{abstract}

1. Introduction. The expression "special symmetries" in the title refers to suitable subgroups of $\mathcal{G}(\mathrm{X})=\{T: \mathrm{X} \rightarrow \mathrm{X}: T$ an isometric automorphism $\}$ where $\mathrm{X}$ is a real Banach space. We denote the closed unit ball of $\mathrm{X}$ by $\mathbf{B}_{\mathrm{X}}$ and the unit sphere by $\mathbf{S}_{\mathrm{X}}$. The orbit of $x \in \mathbf{S}_{\mathrm{X}}$ with respect to a family $\mathcal{F} \subset L(\mathrm{X})$ is given by $\mathcal{F}(x)=\{T(x): T \in \mathcal{F}\}$. An inner product $(\cdot \mid \cdot): \mathrm{X} \times \mathrm{X} \rightarrow \mathbb{R}$ is said to be invariant with respect to $\mathcal{F}$ if $(T(x) \mid T(y))=(x \mid y)$ for each $x, y \in \mathrm{X}, T \in \mathcal{F}$. The concept of an invariant inner product is an important tool applied frequently in this article. We say that $\mathrm{X}$ is transitive, almost transitive or convex-transitive with respect to $\mathcal{F}$ if $\mathcal{F}(x)=\mathbf{S}_{\mathrm{X}}, \overline{\mathcal{F}(x)}=\mathbf{S}_{\mathrm{X}}$ or $\overline{\operatorname{conv}}(\mathcal{F}(x))=\mathbf{B}_{\mathrm{X}}$, respectively, for all $x \in \mathbf{S}_{\mathrm{X}}$. If $\mathcal{F}=\mathcal{G}(\mathrm{X})$ above, then we will omit mentioning it. This article can be regarded as a part of the field generated around the well-known open Banach-Mazur rotation problem, which asks whether each transitive separable Banach space is isometrically a Hilbert space. See [3] for an exposition of the topic.

In [5] F. Cabello Sánchez studied the subgroup

$$
\mathcal{G}_{F}=\{T \in \mathcal{G}(\mathrm{X}): \operatorname{Rank}(T-\mathrm{Id})<\infty\}
$$

consisting of the finite-dimensional perturbations of the identity. There a classical result appearing in [1, 11] is applied, namely, that each finite-dimen-

2010 Mathematics Subject Classification: Primary 46C15, 43A35; Secondary 46B08, $43 \mathrm{~A} 07$.

Key words and phrases: amenable, finite-dimensional isometric perturbations of the identity, isometry group, Mazur's problem, rotation. 
sional Banach space admits an invariant inner product. This motivated the work in [5], where an elegant proof was presented for the following result:

THEOREM 1.1. If the norm of $\mathrm{X}$ is transitive with respect to $\mathcal{G}_{F}$, then $\mathrm{X}$ is isometric to a Hilbert space.

Cabello raised the question whether this result can be extended to the almost transitive setting. It turns out that the answer is affirmative under the additional assumption that $\mathrm{X}$ is isomorphic to a Hilbert space:

TheOREM 1.2. Let $\mathrm{X}$ be a Banach space isomorphic to a Hilbert space. Then $\mathrm{X}$ is convex-transitive with respect to $\mathcal{G}_{F}$ if and only if $\mathrm{X}$ is isometric to a Hilbert space.

This paper is also motivated by the following problems posed in [4, 5]:

- Is an almost transitive Banach space isometric to a Hilbert space if it is isomorphic to one?

- Find ideals $J \subset L(\mathrm{X})$ (with $F \subset J$ ) for which Theorem 1.1 remains true if the condition $T-\operatorname{Id} \in F$ is replaced by $T-\operatorname{Id} \in J$ (here $F$ is the ideal of finite-rank operators).

Questions of this type are treated in what follows, and we will also show that the existence of an invariant inner product on $\mathrm{X}$ follows from the existence of an invariant inner product for each finitely generated subgroup of $\mathcal{G}(\mathrm{X})$ (see Theorem 2.2).

1.1. Preliminaries. We refer to [3, 7], [9], [10], [13] and [14] for some background information. Recall that a norm $\|\cdot\|$ on $\mathrm{X}$ is maximal if $\mathcal{G}_{(\mathrm{X},\|\cdot\|)} \subset$ $\mathcal{G}_{(\mathrm{X},\|\cdot\| \cdot \|)}$ for an equivalent norm $\left\||\cdot \|| \mid\right.$ implies that $\mathcal{G}_{(\mathrm{X},\|\cdot\|)}=\mathcal{G}_{(\mathrm{X},\|\cdot\| \cdot \|)}$. If $\mathrm{X}$ is convex-transitive, then the norm of $\mathrm{X}$ is maximal (see [6]). We denote by Aut $(\mathrm{X})$ the group of isomorphisms $T: \mathrm{X} \rightarrow \mathrm{X}$.

Given a topological group $G$ we denote by $\operatorname{UCB}(G)$ the space of uniformly continuous bounded functions on $G$. Here we consider the uniform structure $\Phi_{G}$ of $G$ as being generated by a basis of entourages of the diagonal having the form

$$
W=\left\{(g, h) \in G \times G: g h^{-1}, g^{-1} h \in V\right\},
$$

where $V$ runs over a neighbourhood basis of $e$ in $G$. The space $\operatorname{UCB}(G)$ is endowed with the $\|\cdot\|_{\infty}$-norm.

For convenience we isolate the following condition: Suppose that there is a positive functional $F \in \operatorname{UCB}(G)^{*}$ with $\|F\|=1$ such that

$$
F(f(\cdot g))=F(f(\cdot)) \quad \text { for all } f \in \mathrm{UCB}(G), g \in G .
$$

This type of condition can be viewed as a weaker version of amenability of $G$ (see [12]). We note that the rotation group of $L^{p}$ with the strong operator topology is extremely amenable for $1 \leq p<\infty$ (see [9]). 
Recall that the product topology of $\mathrm{X}^{\mathrm{X}}$ inherited by $L(\mathrm{X})$ is called the strong operator topology (SOT).

We often consider subgroups $\mathcal{G} \subset \mathcal{G}(\mathrm{X})$ which enjoy the following property:

(*) Given $n \in \mathbb{N}, T_{1}, \ldots, T_{n} \in \mathcal{G}$ and a finite-codimensional subspace $\mathrm{Z} \subset \mathrm{X}$ there exists a finite-codimensional subspace $\mathrm{Y} \subset \mathrm{Z}$ such that $T_{1}(\mathrm{Y})=\cdots=T_{n}(\mathrm{Y})=\mathrm{Y}$.

Clearly $\mathcal{G}_{F}$ is an example of a subgroup of $\mathcal{G}(\mathrm{X})$ satisfying $(*)$.

It is easy to see that if $\mathrm{H}$ is a Hilbert space, then $\mathcal{G}_{F} \subset \mathcal{G}(\mathrm{H})$ is dense in $\mathcal{G}(\mathrm{H})$ in the topology of uniform convergence on compact sets. On the other hand, given a Banach space $\mathrm{X}$ the group $\mathcal{G}(\mathrm{X})$ is SOT-closed in $\operatorname{Aut}(\mathrm{X})$.

\section{Results}

Theorem 2.1. Let $\mathrm{X}$ be a maximally normed Banach space which is isomorphic to a Hilbert space. Suppose that $\mathcal{G}(\mathrm{X})$ endowed with the strong operator topology is amenable in the sense of condition (1.2). Then $\mathrm{X}$ is isometrically isomorphic to a Hilbert space.

Proof. We may assume without loss of generality that $(\mathrm{X},\|\cdot\|)$ and $(\mathrm{X},|\cdot|)$ are isomorphic via the identical mapping, where $|\cdot|$ is a norm induced by an inner product $(\cdot \mid \cdot)$ on $\mathrm{X}$. We denote by $\mathcal{G}(\mathrm{X})=\mathcal{G}_{(\mathrm{X},\|\cdot\|)}$ and $\mathcal{G}_{(\mathrm{X},|\cdot|)}$ the corresponding rotation groups, and these are regarded with the strong operator topology. Recall that $\Phi_{\mathcal{G}(\mathrm{X})}$ is the natural uniformity given by the group $(\mathcal{G}(\mathrm{X}), \mathrm{SOT})$ applied to $(1.1)$.

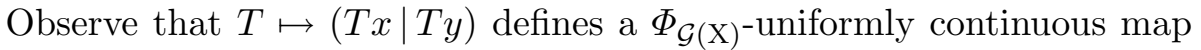
$\mathcal{G}(\mathrm{X}) \rightarrow \mathbb{R}$ for each $x, y \in \mathrm{X}$. Indeed, this map is obtained by composing the $\Phi_{\mathcal{G}(\mathrm{X})^{-}}\|\cdot\|_{\mathrm{X} \oplus_{2} \mathrm{X}}$ uniformly continuous map $\mathcal{G}(\mathrm{X}) \rightarrow \mathrm{X} \oplus_{2} \mathrm{X}, T \mapsto(T x, T y)$, and the map $(T x, T y) \mapsto(T x \mid T y)$, which is $\|\cdot\|_{\mathrm{X} \oplus_{2} \mathrm{X} \text {-uniformly continuous }}$ as $\|\cdot\| \sim|\cdot|$. To check that $T \mapsto(T x, T y)$ is uniformly continuous, first consider a standard entourage

$$
E=\left\{\left(x_{1}, y_{1}, x_{2}, y_{2}\right) \in \mathrm{X} \oplus_{2} \mathrm{X} \times \mathrm{X} \oplus_{2} \mathrm{X}:\left\|\left(x_{1}, y_{1}\right)-\left(x_{2}, y_{2}\right)\right\|_{\mathrm{X} \oplus_{2} \mathrm{X}}<\epsilon\right\}
$$

for some $\epsilon>0$. The preimage of this is

$$
\begin{aligned}
\{(R, S) & \left.\in \mathcal{G}(\mathrm{X}) \times \mathcal{G}(\mathrm{X}):\|(R x, R y)-(S x, S y)\|_{\mathrm{X} \oplus_{2} \mathrm{X}}<\epsilon\right\} \\
& \supset\{(R, S) \in \mathcal{G}(\mathrm{X}) \times \mathcal{G}(\mathrm{X}):\|T x-S x\|,\|T y-S y\|<\epsilon / 2\} \\
= & \left\{(R, S) \in \mathcal{G}(\mathrm{X}) \times \mathcal{G}(\mathrm{X}):\left\|x-T^{-1} S x\right\|,\left\|y-T^{-1} S y\right\|<\epsilon / 2\right\} .
\end{aligned}
$$

Hence it suffices to pick $V=\{R \in \mathcal{G}(\mathrm{X}):\|x-R x\|,\|y-R y\|<\epsilon / 2\}$ in (1.1) to find an entourage of $\Phi_{\mathcal{G}(\mathrm{X})}$ in the preimage of $E$. We deduce that $T \mapsto(T x, T y)$ is $\Phi_{\mathcal{G}(\mathrm{X})}$-uniformly continuous. 
According to the assumptions there is $F \in \mathrm{UCB}(\mathcal{G}(\mathrm{X}))^{*}$ with $\|F\|=1$ such that $F(f(\cdot g))=F(f(\cdot))$ for $f \in \mathrm{UCB}(\mathcal{G}(\mathrm{X}))$ and $g \in \mathcal{G}(\mathrm{X})$. For each $x, y \in \mathrm{X}$ we put

$$
[x \mid y]=F\left(\{(g(x) \mid g(y))\}_{g \in \mathcal{G}(\mathrm{X})}\right) .
$$

This definition is sensible, since $g \mapsto(g(x) \mid g(y))$ defines an element in $\mathrm{UCB}(\mathcal{G}(\mathrm{X}))$ for each $x, y \in \mathrm{X}$. We claim that $[\cdot \mid \cdot]$ defines an inner product on $\mathrm{X}$ such that $\|x\| \| \doteq \sqrt{[x \mid x]}$ is equivalent to $\|\cdot\|$. Indeed, first note that $[\cdot \mid \cdot]:(\mathrm{X},\|\cdot\|) \oplus_{2}(\mathrm{X},\|\cdot\|) \rightarrow \mathbb{R}$ is defined and bounded, since $(\cdot \mid \cdot)$ : $(\mathrm{X},\|\cdot\|) \oplus_{2}(\mathrm{X},\|\cdot\|) \rightarrow \mathbb{R}$ is bounded and $\|F\|=1$. By using the bilinearity of $(\cdot \mid \cdot)$ and the linearity of $F$ we see that $[\cdot \mid \cdot]$ is bilinear. Let $C \geq 1$ be such that $C^{-2}\|\cdot\|^{2} \leq|\cdot|^{2} \leq C^{2}\|\cdot\|^{2}$. Since $F$ is positive and norm-1, we get

$$
\begin{aligned}
C^{-2}\|x\|^{2} & =\inf _{g} C^{-2}\|g(x)\|^{2} \leq F\left(\{(g(x) \mid g(x))\}_{g \in \mathcal{G}(\mathrm{X})}\right) \\
& \leq \sup _{g} C^{2}\|g(x)\|=C^{2}\|x\|,
\end{aligned}
$$

where $x \in \mathrm{X}$ and the supremum and infimum are taken over $\mathcal{G}(\mathrm{X})$. This means that $[\cdot \mid \cdot]$ is an inner product on $\mathrm{X}$ such that $\|\mid \cdot\|$ is equivalent to $\|\cdot\|$.

Observe that

$$
[h(x) \mid h(y)]=F\left(\{(g h(x) \mid g h(y))\}_{g \in \mathcal{G}(\mathrm{X})}\right)=F\left(\{(g(x) \mid g(y))\}_{g \in \mathcal{G}(\mathrm{X})}\right)=[x \mid y]
$$

for each $h \in \mathcal{G}(\mathrm{X})$. The maximality of the norm of $(\mathrm{X},\|\cdot\|)$ implies that $\mathcal{G}_{(\mathrm{X},\|\cdot\|)}=\mathcal{G}_{(\mathrm{X},\|\cdot \cdot\|)}$. The proof is completed by a standard argument using the fact that $(\mathrm{X},\||\cdot|\|)$ is transitive.

Suppose that $\mathrm{X}$ is a Banach space with two equivalent norms $\|\cdot\|$ and $\left\|\left|\|\mid\|\right.\right.$ such that the group $\mathcal{G}$ generated by $\mathcal{G}_{(\mathrm{X},\|\cdot\|)} \cup \mathcal{G}_{(\mathrm{X},\|\cdot\| \cdot \|)}$ is operator norm bounded. Then there is one more equivalent norm \|\| $\mid \cdot\|\|$ on $\mathrm{X}$ given by \|\|$x \mid\|\|=\sup _{g \in \mathcal{G}}\|g(x)\|$ and this is $\mathcal{G}$-invariant. Consequently, if the norms $\|\cdot\|$ and $\||\cdot|\|$ are additionally maximal (resp. convex-transitive), then $\mathcal{G}_{(\mathrm{X},\|\cdot\|)}=\mathcal{G}_{(\mathrm{X},\|\cdot\| \cdot \|)}($ resp. $\|\cdot\|=c|\|\cdot\||$ for some constant $c>0$ ).

The argument employed in the proof of [5, Lemma 2] can be modified to obtain the following dichotomy regarding the existence of invariant inner products.

Theorem 2.2. Let $\mathrm{X}$ be a Banach space and $C \geq 1$. Suppose that for each $n \in \mathbb{N}$ and $T_{1}, \ldots, T_{n} \in \mathcal{G}(\mathrm{X})$ there exists an inner product $(\cdot \mid \cdot)_{*}$ : $\mathrm{X} \times \mathrm{X} \rightarrow \mathbb{R}$ invariant under the rotations $T_{1}, \ldots, T_{n}$ such that $C^{-2}\|x\|^{2} \leq$ $(x \mid x)_{*} \leq C^{2}\|x\|^{2}$ for each $x \in \mathrm{X}$. Then there is already an inner product $(\cdot \mid \cdot)_{\mathrm{X}}: \mathrm{X} \times \mathrm{X} \rightarrow \mathbb{R}$ which is invariant under $\mathcal{G}(\mathrm{X})$ and satisfies $C^{-2}\|x\|^{2} \leq$ $(x \mid x)_{\mathrm{X}} \leq C^{2}\|x\|^{2}$ for $x \in \mathrm{X}$.

Proof. We may assume without loss of generality that $\mathcal{G}(\mathrm{X})$ is not finitely generated. Let $\mathcal{N}$ be the net of finitely generated subgroups of $\mathcal{G}(\mathrm{X})$ ordered 
by inclusion. By the assumptions we may assign to each $\gamma \in \mathcal{N}$ an inner product $(\cdot \mid \cdot)_{\gamma}: \mathrm{X} \times \mathrm{X} \rightarrow \mathbb{R}$ invariant under $\gamma$ and satisfying

$$
C^{-1}\|x\|^{2} \leq(x \mid x)_{\gamma} \leq C\|x\|^{2} \quad \text { for } x \in \mathrm{X} .
$$

Observe that the sets $\{\gamma \in \mathcal{N}: \delta \subset \gamma\}$, where $\delta \in \mathcal{N}$, form a filter base of a filter $\mathcal{F}$ on $\mathcal{N}$. Let us extend $\mathcal{F}$ to an ultrafilter $\mathcal{U}$ on $\mathcal{N}$. Note that $\mathcal{U}$ is non-principal, since for each $\eta \in \mathcal{N}$ there is $\delta \in \mathcal{N}$ with $\eta \subsetneq \delta$ such that $\eta \notin\{\gamma \in \mathcal{N}: \delta \subset \gamma\} \in \mathcal{U}$.

Define $B: \mathrm{X} \times \mathrm{X} \rightarrow \mathbb{R}^{\mathcal{N}}$ by setting $B(x, y)=\left\{(x \mid y)_{\gamma}\right\}_{\gamma \in \mathcal{N}}$ for $x, y \in \mathrm{X}$. We will consider $\mathbb{R}^{\mathcal{N}}$ equipped with the usual pointwise linear structure. Then $B$ becomes a symmetric and bilinear map. Moreover, $B(x, x) \geq 0$ pointwise for $x \in \mathrm{X}$. Put $\vec{B}: \mathrm{X} \times \mathrm{X} \rightarrow \mathbb{R}, \vec{B}(x, y)=\lim _{\mathcal{U}} B(x, y)$ for $x, y \in \mathrm{X}$. Indeed, the above limit exists and is finite for all $x, y \in \mathrm{X}$, since

$$
(x \mid y)_{\gamma} \leq \sqrt{(x \mid x)_{\gamma}(y \mid y)_{\gamma}} \leq C^{2}\|x\|\|y\| \quad \text { for all } \gamma \in \mathcal{N}, x, y \in \mathrm{X} .
$$

Moreover, similarly we get $C^{-2}\|x\|^{2} \leq \vec{B}(x, x) \leq C^{2}\|x\|^{2}$ for all $x \in \mathrm{X}$. It follows that $\vec{B}$ is an inner product on $\mathrm{X}$.

Observe that for all $T \in \mathcal{G}(\mathrm{X})$ and $x, y \in \mathrm{X}$ we have

$$
\left\{\gamma \in \mathcal{N}:(T x \mid T y)_{\gamma}=(x \mid y)_{\gamma}\right\} \supset\{\gamma \in \mathcal{N}: T \in \gamma\} \in \mathcal{F} \subset \mathcal{U} .
$$

Hence $\vec{B}(T x, T y)=\vec{B}(x, y)$ for $T \in \mathcal{G}(\mathrm{X})$ and $x, y \in \mathrm{X}$. Consequently, $\vec{B}$ is the required inner product.

It is not known if an almost transitive Banach space isomorphic to a Hilbert space is in fact isometric to a Hilbert space (see [4]). The following consequence of Theorem 2.2 provides a partial answer to this problem.

Corollary 2.3. Let $\mathrm{X}$ be a maximally normed Banach space, $\mathrm{H}$ a Hilbert space and $C \geq 1$. Suppose that for any $n \in \mathbb{N}$ and $T_{1}, \ldots, T_{n} \in \mathcal{G}(\mathrm{X})$ there exists an isomorphism $\phi: \mathrm{X} \rightarrow \mathrm{H}$ such that $\max \left(\|\phi\|,\left\|\phi^{-1}\right\|\right) \leq C$ and $\|\phi(x)\|=\left\|\phi\left(T_{i} x\right)\right\|$ for all $x \in \mathrm{X}$ and $i \in\{1, \ldots, n\}$. Then $\mathrm{X}$ is already isometric to $\mathrm{H}$.

Proof. By putting $(x \mid y)_{*}=(\phi(x) \mid \phi(y))_{\mathrm{H}}$ for each $T_{1}, \ldots, T_{n}$ we obtain the assumptions of Theorem 2.2 . Let $(\cdot \mid \cdot)_{\mathrm{X}}: \mathrm{X} \times \mathrm{X} \rightarrow \mathbb{R}$ be the resulting inner product. Then $\mathrm{X}$ endowed with the norm $\|x\| \| \doteq \sqrt{(x \mid x)_{\mathrm{X}}}$ is transitive being a Hilbert space. Since $\mathrm{X}$ is maximally normed, we get $\mathcal{G}_{(\mathrm{X},\|\cdot\|)}=\mathcal{G}_{(\mathrm{X},\|\cdot\|)}$. Thus $\mathrm{X}$ is transitive. It follows that $\|\cdot\|=c\|\| \cdot \|$ for some $c>0$, and hence $\mathrm{X}$ is a Hilbert space.

Theorem 2.4. Let $(\mathrm{X},\|\cdot\|)$ be a Banach space, $\left(\mathrm{H},(\cdot \mid \cdot)_{\mathrm{H}}\right)$ an inner product space, $\mathcal{G} \subset \mathcal{G}(\mathrm{X})$ a subgroup satisfying $(*)$, and $S: \mathrm{X} \rightarrow \mathrm{H}$ an isomorphism. Then there exists an inner product $(\cdot \mid \cdot)_{\mathrm{X}}$ on $\mathrm{X}$ such that 
(1) $\left\|S^{-1}\right\|^{-2}\|x\|^{2} \leq(x \mid x)_{\mathrm{X}} \leq\|S\|^{2}\|x\|^{2}$ for $x \in \mathrm{X}$.

(2) $(T x \mid T y)_{\mathrm{X}}=(x \mid y)_{\mathrm{X}}$ for $x, y \in \mathrm{X}$ and $T \in \overline{\mathcal{G}}^{\mathrm{SOT}} \subset L(\mathrm{X})$.

Proof. It suffices to find $(\cdot \mid \cdot)_{\mathrm{X}}$ which satisfies conclusions (1) and (2) for merely $T \in \mathcal{G}$. Indeed, given $T \in \overline{\mathcal{G}}^{\mathrm{SOT}}$ and $x, y \in \mathrm{X}$ there is a sequence $\left(T_{n}\right) \subset \mathcal{G}$ such that $T_{n}(x) \rightarrow T(x)$ and $T_{n}(y) \rightarrow T(y)$ as $n \rightarrow \infty$. This yields

$$
(T(x) \mid T(y))_{\mathrm{X}}-(x \mid y)_{\mathrm{X}}=\lim _{n \rightarrow \infty}\left(\left(T_{n}(x) \mid T_{n}(y)\right)_{\mathrm{X}}-(x \mid y)_{\mathrm{X}}\right)=0
$$

by using the $\mathcal{G}$-invariance and the $\|\cdot\|$-continuity of $(\cdot \mid \cdot)_{\mathrm{X}}$.

Let $\mathcal{M}$ be the set of all pairs $(E, G)$ where $E \subset \mathrm{X}$ is a finite-codimensional subspace and $G \subset \mathcal{G}$ is a finitely generated subgroup such that $T(E)=E$ for $T \in G$.

From the definition of $\mathcal{G}$ we know that $\bigcup_{(E, G) \in \mathcal{M}} G=\mathcal{G}$ and $\bigcap_{(E, G) \in \mathcal{M}} E$ $=\{0\}$. We equip $\mathcal{M}$ with the partial order $\leq$ defined as follows: $\left(E_{1}, G_{1}\right) \leq$ $\left(E_{2}, G_{2}\right)$ if $E_{1} \supset E_{2}$ and $G_{1} \subset G_{2}$. So, $(\mathcal{M}, \leq)$ is a directed set.

Suppose that $\mathrm{Y} \subset \mathrm{H}$ is a subspace of a Hilbert space and $\mathrm{H} / \mathrm{Y}$ is the corresponding quotient space. Then there exists a natural inner product on $\mathrm{H} / \mathrm{Y}$, namely

$$
\left(\widehat{x}^{\mathrm{Y}} \mid \widehat{y}^{\mathrm{Y}}\right)_{\mathrm{H} / \mathrm{Y}}=\left(x-P_{\mathrm{Y}} x \mid y-P_{\mathrm{Y}} y\right)_{\mathrm{H}}, \quad x, y \in \mathrm{H},
$$

where $\widehat{x}^{Y}=x+\mathrm{Y}, \widehat{y}^{\mathrm{Y}}=y+\mathrm{Y}$ and $P_{\mathrm{Y}}: \mathrm{X} \rightarrow \mathrm{Y}$ is the orthogonal projection onto Y.

Given $(E, G) \in \mathcal{M}$ we find that $T(E)=E$ for $T \in G$ and hence the mapping $\widehat{T}_{E}: \mathrm{X} / E \rightarrow \mathrm{X} / E$ given by $\widehat{T}_{E}\left(\widehat{x}^{E}\right)=T(x+E)$ defines a rotation on $\mathrm{X} / E$ for $T \in G$. Indeed, $\left\|\widehat{x}^{E}\right\|_{\mathrm{X} / E}=\operatorname{dist}(x, E)$ and $\operatorname{dist}(T(x), E)=$ $\operatorname{dist}(x, E)$, as $T(E)=E$. Now, since $\mathrm{X} / E$ is finite-dimensional, the rotation group $\mathcal{G}_{\mathrm{X} / E}$ is compact in the operator norm topology.

For each $(E, G) \in \mathcal{M}$ we define a map $\widehat{S}_{E}: \mathrm{X} / E \rightarrow \mathrm{H} / S(E)$ by $\widehat{S}_{E}\left(\widehat{x}^{E}\right)=$ $S(x+E)$. It is easy to see that

$$
\begin{aligned}
& \left\|S^{-1}\right\|^{-2}\left\|\widehat{x}^{E}\right\|_{\mathrm{X} / E}^{2} \leq\left(\widehat{S}_{E}\left(\widehat{x}^{E}\right) \mid \widehat{S}_{E}\left(\widehat{x}^{E}\right)\right)_{\mathrm{H} / S(E)}, \\
& \left(\widehat{S}_{E}\left(\widehat{x}^{E}\right) \mid \widehat{S}_{E}\left(\widehat{y}^{E}\right)\right)_{\mathrm{H} / S(E)} \leq\|S\|^{2}\left\|\widehat{x}^{E}\right\|_{\mathrm{X} / E}\left\|\widehat{y}^{E}\right\|_{\mathrm{X} / E}
\end{aligned}
$$

for $x, y \in \mathrm{X}$. Consider $\mathbb{R}^{\mathcal{M}}$ with the pointwise linear structure. Define a map $B: \mathrm{X} \times \mathrm{X} \rightarrow \mathbb{R}^{\mathcal{M}}$ by

$$
B(x, y)(E, G)=\int_{\mathcal{G}_{\mathrm{X} / E}}\left(\widehat{S}_{E}\left(\tau \widehat{x}^{E}\right) \mid \widehat{S}_{E}\left(\tau \widehat{y}^{E}\right)\right)_{\mathrm{H} / S(E)} d \tau .
$$

Above $\int_{\mathcal{G}_{\mathrm{X} / E}}$ is the invariant Haar integral over the compact group $\mathcal{G}_{\mathrm{X} / E}$. The invariance of the integral yields $B(T x, T y)(E, G)=B(x, y)(E, G)$ for $x, y \in \mathrm{X},(E, G) \in \mathcal{M}$ and $T \in G$. By using (2.1) and the basic properties 
of the integral we obtain

$$
\begin{aligned}
& \left\|S^{-1}\right\|^{-2}\left\|\widehat{x}^{E}\right\|_{\mathrm{X} / E}^{2} \leq B(x, x)(E, G), \\
& B(x, y)(E, G) \leq\|S\|^{2}\left\|\widehat{x}^{E}\right\|_{\mathrm{X} / E}\left\|\widehat{y}^{E}\right\|_{\mathrm{X} / E}
\end{aligned}
$$

for $x, y \in \mathrm{X}$ and $(E, G) \in \mathcal{M}$.

The family $\{\{\gamma \in \mathcal{M}: \gamma \geq \eta\}\}_{\eta \in \mathcal{M}}$ is a filter base on $\mathcal{M}$. Let $\mathcal{U}$ be a non-principal ultrafilter extending $\{\{\gamma \in \mathcal{M}: \gamma \geq \eta\}\}_{\eta \in \mathcal{M}}$. Put $(x \mid y)_{\mathrm{X}}=$ $\lim _{\mathcal{U}} B(x, y)$ for $x, y \in \mathrm{X}$. It is easy to see that $(\cdot \mid \cdot)_{\mathrm{X}}$ is a bilinear mapping.

According to 2.2 we obtain $(x \mid y)_{\mathrm{X}} \leq\|S\|^{2}\|x\|_{\mathrm{X}}\|y\|_{\mathrm{X}}$. Next, we aim to verify that $\left\|S^{-1}\right\|^{-2}\|x\|_{\mathrm{X}}^{2} \leq(x \mid x)_{\mathrm{X}}$. Towards this, we will check that $\sup _{(E, G) \in \mathcal{M}}\left\|\widehat{x}^{E}\right\|_{\mathrm{X} / E}=\|x\|_{\mathrm{X}}$. Fix $x \in \mathbf{S}_{\mathrm{X}}$. Assume to the contrary that $\sup _{(E, G) \in \mathcal{M}}\left\|\widehat{x}^{E}\right\|_{\mathrm{X} / E}=c<1$. Note that $\mathrm{X}$ is reflexive, being isomorphic to $\mathrm{H}$. Thus the ball $x+c \mathbf{B}_{\mathrm{X}}$ is weakly compact. Putting

$$
\{\{y \in E:\|x-y\| \leq C\}\}_{(E, G) \in \mathcal{M}}
$$

defines a net of non-empty closed convex subsets of $x+c \mathbf{B}_{\mathrm{X}}$. This net has a cluster point $z \in x+c \mathbf{B}_{\mathrm{X}}$ according to the weak compactness of $x+c \mathbf{B}_{\mathrm{X}}$. This means that $z \in \bigcap_{(E, G) \in \mathcal{M}} E$, which provides a contradiction, since $z \neq 0$. Consequently, 2.2 yields

$$
\left\|S^{-1}\right\|^{-2}\|x\|_{\mathrm{X}}^{2}=\left\|S^{-1}\right\|^{-2} \lim _{\mathcal{U}}\left\|\widehat{x}^{E}\right\|_{\mathrm{X} / E}^{2} \leq \lim _{\mathcal{U}} B(x, x)=(x \mid x)_{\mathrm{X}} .
$$

Finally, we claim that $(T x \mid T y)_{\mathrm{X}}=(x \mid y)_{\mathrm{X}}$ for $x, y \in \mathrm{X}$ and $T \in \mathcal{G}$. Indeed, pick $T \in \mathcal{G}$ and $x, y \in \mathrm{X}$. Then

$$
\begin{aligned}
\{(E, G) \in \mathcal{M}: B(T(x), T(y))(E, G)= & B(x, y)(E, G)\} \\
& \supset\{(E, G) \in \mathcal{M}: T \in G\} \in \mathcal{U},
\end{aligned}
$$

so that $\lim _{\mathcal{U}}(B(T x, T y)-B(x, y))=0$.

COROllary 2.5. Let X be a maximally normed space $\mathrm{X}$ isomorphic to a Hilbert space. Suppose that there is a subgroup $\mathcal{G} \subset \mathcal{G}(\mathrm{X})$ which satisfies (*) and $\mathcal{G}(\mathrm{X}) \subset \overline{\mathcal{G}}^{\mathrm{SOT}}$. Then $\mathrm{X}$ is isometrically a Hilbert space.

In Theorem 2.4 the isomorphism $S$ was exploited in order to give bounds for the resulting inner product $(\cdot \mid \cdot)_{\mathrm{X}}$. In [5] a different approach was taken: the analogous construction was suitably normalized by using a special point $x_{0}$. By suitably combining the arguments in [5] and in the proof of Theorem 2.4 we obtain the following result.

THEOREM 2.6. Let $\mathrm{X}$ be a Banach space transitive with respect to a subgroup $\mathcal{G} \subset \mathcal{G}(\mathrm{X})$ which satisfies $(*)$. Then $\mathrm{X}$ is isometric to a Hilbert space. 
Theorem 1.2 is an immediate consequence of the following result. This result implies that $\mathrm{X}$ must in particular be almost transitive, and we note that there exists an alternative route to this fact, since spaces both convextransitive and superreflexive are additionally almost transitive (see e.g. [8]).

TheOREM 2.7. Let X be a Banach space isomorphic to a Hilbert space and suppose $\mathcal{G} \subset \mathcal{G}(\mathrm{X})$ is a subgroup which satisfies $(*)$ and $\mathcal{G}_{F} \subset \mathcal{G}$. Then $\mathrm{X}$ is convex-transitive with respect to $\overline{\mathcal{G}}^{\mathrm{SOT}} \subset L(\mathrm{X})$ if and only if $\mathrm{X}$ is isometric to a Hilbert space.

Proof. First note that a Hilbert space is transitive, in particular convextransitive, and that $\mathcal{G}_{F} \subset \mathcal{G}(\mathrm{H})$ is SOT-dense in $\mathcal{G}(\mathrm{H})$, so that the "if" direction is clear.

Since $\mathrm{X}$ is isomorphic to a Hilbert space, we may apply Theorem 2.4 to obtain a $\overline{\mathcal{G}}^{\mathrm{SOT}}$-invariant inner product $(\cdot \mid \cdot)_{\mathrm{X}}$ on $\mathrm{X}$ such that $\left.\|x\|\right|^{2}=(x \mid x)_{\mathrm{X}}$ defines a norm equivalent with $\|\cdot\|_{\mathrm{X}}$. Clearly \|\|$\cdot \|$ is $\overline{\mathcal{G}}^{\mathrm{SOT}}$-invariant as well. By rescaling $|\|\cdot \mid\|$ we may assume without loss of generality that $\|\cdot\| \mathrm{x} \leq\||| \cdot \mid\|$ and $\sup _{y \in \mathbf{S}_{(\mathrm{X},\|\cdot\| \cdot \|)}}\|y\|_{\mathrm{X}}=1$. Put $C=\{x \in \mathrm{X}:\|x\| \leq 1\}$.

Fix $x \in \mathbf{S}_{\left(\mathrm{X},\|\cdot\|_{\mathrm{X}}\right)}$ and $\epsilon>0$. Let $y \in \mathbf{S}_{(\mathrm{X},\|\cdot\| \cdot \|)}$ be such that $\|y\|_{\mathrm{X}}>$ $1-\epsilon / 2$. Since $\left(\mathrm{X},\|\cdot\|_{\mathrm{X}}\right)$ is convex-transitive with respect to $\overline{\mathcal{G}}^{\mathrm{SOT}}$, we see that $(1-\epsilon / 2) x \in \overline{\mathrm{conv}}\|\cdot\|_{\mathrm{x}}\left(\left\{T(y): T \in \overline{\mathcal{G}}^{\mathrm{SOT}}\right\}\right)$. Since the norms $\|\cdot\| \|$ and $\|\cdot\|_{\mathrm{X}}$ are equivalent we deduce that there is a convex combination $\sum a_{n} T_{n}(y) \in$ $\operatorname{conv}\left(\left\{T(y): T \in \mathcal{G}_{F}\right\}\right)$ such that $\left\|(1-\epsilon / 2) x-\sum a_{n} T_{n}(y) \mid\right\|<\epsilon / 2$. By noting that ||$\sum a_{n} T_{n}(y)|| \leq \sum a_{n}|| T_{n}(y) \|||$ we obtain $\sup _{T \in \overline{\mathcal{G}}^{\text {оОт }}}|||T(y)| \| \geq$ $\||| x|| \mid-\epsilon$. Hence $\|y\|\left|\geq\left\|\left|\|\mid\|-\epsilon\right.\right.\right.$ by using the $\overline{\mathcal{G}}^{\mathrm{SOT}}$-invariance of $\||\cdot|\|$. Since $\epsilon$ was arbitrary and $\||x|\| \geq 1$, we deduce that $|\|x \mid\|=1$, and it follows that $\|\cdot\|_{\mathrm{X}}=|\|\cdot \mid\|$.

Finally, we will take a different approach and characterize the Hilbert spaces in terms of the subgroup of rotations that fix a given 1-dimensional subspace, rather than a finite-codimensional subspace.

Proposition 2.8. Let X be an almost transitive Banach space. Suppose that there exists $z_{0} \in \mathbf{S}_{\mathrm{X}}$ such that for any $\epsilon>0$ and $x, y \in \mathbf{S}_{\mathrm{X}}$ with $\operatorname{dist}\left(x,\left[z_{0}\right]\right)=\operatorname{dist}\left(y,\left[z_{0}\right]\right)=1$, there is $T \in \mathcal{G}(\mathrm{X})$ with $\left\|T\left(z_{0}\right)-z_{0}\right\|<\epsilon$ and $\|T(x)-y\|<\epsilon$. Then $\mathrm{X}$ is isometric to an inner product space.

Proof. It is well-known (see e.g. Corollary 2.42 and Diagram I in [3, p. 22], or Proposition 9.6.1 and discussion in [13]) that almost transitive finite-dimensional spaces are isometric to Hilbert spaces. Hence we may concentrate on the case $\operatorname{dim}(\mathrm{X}) \geq 3$. Let $A, B \subset \mathrm{X}$ be 2 -dimensional subspaces such that $z_{0} \in A$. Recall the classical result that a Banach space is isometric to a Hilbert space if and only if any couple of 2-dimensional subspaces are 
mutually isometric (see [2]). Thus, in order to establish the claim, it suffices to verify that the subspaces $A$ and $B$ are isometric.

Fix $0<\epsilon<1, x \in \mathbf{S}_{\mathrm{X}} \cap A$ such that $\operatorname{dist}\left(x,\left[z_{0}\right]\right)=1$ and $w \in \mathbf{S}_{\mathrm{X}} \cap B$. Let $f \in \mathbf{S}_{\mathrm{X}}$ be such that $f(w)=1$.

Since $\mathrm{X}$ is almost transitive, there is $T_{1} \in \mathcal{G}(\mathrm{X})$ such that $\left\|T_{1}(w)-z_{0}\right\|<$ $\epsilon / 4$. Define a linear operator $S: \mathrm{X} \rightarrow \mathrm{X}$ by $S(v)=T_{1}(v)+f(v)\left(z_{0}-T_{1}(w)\right)$ for $v \in \mathrm{X}$ and note that $S(w)=z_{0}$. Observe that $S$ is an isomorphism, since $\left\|T_{1}-S_{1}\right\|<\epsilon / 4$. Pick $y \in \mathbf{S}_{\mathrm{X}} \cap S(B)$ such that $\operatorname{dist}\left(y,\left[z_{0}\right]\right)=1$. According to the assumptions there is $T_{2} \in \mathcal{G}(\mathrm{X})$ such that

$$
\max \left(\left\|T_{2}\left(z_{0}\right)-z_{0}\right\|,\left\|T_{2}(y)-x\right\|\right)<\epsilon / 4 \text {. }
$$

Let $g, h \in 2 \mathbf{B}_{\mathrm{X}^{*}}$ be such that $g\left(z_{0}\right)=h(y)=1, y \in \operatorname{Ker}(g)$ and $z_{0} \in \operatorname{Ker}(h)$. Define a linear operator $U: \mathrm{X} \rightarrow \mathrm{X}$ by

$$
U(v)=T_{2}(v)+g(v)\left(z_{0}-T_{2}\left(z_{0}\right)\right)+h(v)\left(x-T_{2}(y)\right) \quad \text { for } v \in \mathrm{X} .
$$

Note that $U\left(z_{0}\right)=z_{0}$ and $U(y)=x$. Moreover, $\left\|T_{2}-U\right\|<\epsilon$, so that $U$ is an isomorphism. Observe that $U \circ S$ maps $B$ linearly onto $A$. We conclude that $A$ and $B$ are almost isometric, since $\epsilon$ was arbitrary. Hence, being finite-dimensional spaces, $A$ and $B$ are isometric.

\section{References}

[1] H. Auerbach, Sur les groupes linéaires bornés I, Studia Math. 4 (1934), 113-127.

[2] H. Auerbach, S. Mazur et S. Ulam, Sur une propriété caractéristique de l'ellipsoïde, Monatsh. Math. Phys. 42 (1935), 45-48.

[3] J. Becerra Guerrero and A. Rodríguez-Palacios, Transitivity of the norm on Banach spaces, Extracta Math. 17 (2002), 1-58.

[4] F. Cabello Sánchez, Regards sur le problème des rotations de Mazur, ibid. 12 (1997), 97-116.

[5] -, A theorem on isotropic spaces, Studia Math. 133 (1999), 257-260.

[6] E. R. Cowie, A note on uniquely maximal Banach spaces, Proc. Edinburgh Math. Soc. 26 (1983), 85-87.

[7] M. Fabian, P. Habala, P. Hájek, V. Montesinos Santalucía, J. Pelant and V. Zizler, Functional Analysis and Infinite-Dimensional Geometry, CMS Books Math. 8, Springer, New York, 2001.

[8] C. Finet, Uniform convexity properties of norms on a super-reflexive Banach space, Israel J. Math. 53 (1986), 81-92.

[9] T. Giordano and V. Pestov, Some extremely amenable groups related to operator algebras and ergodic theory, J. Inst. Math. Jussieu 6 (2007), 279-315.

[10] S. Heinrich, Ultraproducts in Banach space theory, J. Reine Angew. Math. 313 (1980), 72-104.

[11] S. Mazur, Quelques propriétés caractéristiques des espaces euclidiens, C. R. Acad. Sci. Paris 207 (1938), 761-764.

[12] N. W. Rickert, Amenable groups and groups with the fixed point property, Trans. Amer. Math. Soc. 127 (1967), 221-232. 
[13] S. Rolewicz, Metric Linear Spaces, 2nd ed., PWN, Warszawa, and Reidel, Dordrecht, 1985.

[14] B. Sims, "Ultra"-techniques in Banach space theory, Queen's Papers Pure Appl. Math. 60, Queen's Univ., Kingston, ON, 1982.

Jarno Talponen

Institute of Mathematics

Aalto University

P.O. Box 1100

FI-00076 Aalto, Finland

E-mail: talponen@cc.hut.fi

Received September 8, 2009

Revised version May 10, 2010 